\title{
Graphs with many copies of a given subgraph
}

\author{
Vladimir Nikiforov \\ Department of Mathematical Sciences, \\ University of Memphis, Memphis TN 38152 \\ vnikifr@memphis.edu \\ Submitted: Oct 8, 2007; Accepted: Mar 3, 2008; Published: Mar 12, 2008 \\ Mathematics Subject Classification: 05C35
}

\begin{abstract}
Let $c>0$, and $H$ be a fixed graph of order $r$. Every graph on $n$ vertices containing at least $c n^{r}$ copies of $H$ contains a "blow-up" of $H$ with $r-1$ vertex classes of size $\left\lfloor c^{r^{2}} \ln n\right\rfloor$ and one vertex class of size greater than $n^{1-c^{r-1}}$. A similar result holds for induced copies of $H$.
\end{abstract}

\section{Main results}

Suppose that a graph $G$ of order $n$ contains $c n^{r}$ copies of a given subgraph $H$ on $r$ vertices. How large "blow-up" of $H$ must $G$ contain? When $H$ is an $r$-clique, this question was answered in [3]: $G$ contains a complete $r$-partite graph with $r-1$ parts of size $\left\lfloor c^{r} \ln n\right\rfloor$ and one part larger than $n^{1-c^{r-1}}$.

The aim of this note is to answer this question for any subgraph $H$.

First we define precisely a "blow-up" of a graph: given a graph $H$ of order $r$ and positive integers $x_{1}, \ldots, x_{r}$, we write $H\left(x_{1}, \ldots, x_{r}\right)$ for the graph obtained by replacing each vertex $u \in V(H)$ with a set $V_{u}$ of size $x_{u}$ and each edge $u v \in E(H)$ with a complete bipartite graph with vertex classes $V_{u}$ and $V_{v}$.

Theorem 1 Let $2 \leq r \leq n,(\ln n)^{-1 / r^{2}} \leq c \leq 1 / 4, H$ be a graph of order $r$, and $G$ be a graph of order $n$. If $G$ contains more than $\mathrm{cn}^{r}$ copies of $H$, then $G$ contains a copy of $H(s, \ldots s, t)$, where $s=\left\lfloor c^{r^{2}} \ln n\right\rfloor$ and $t>n^{1-c^{r-1}}$.

To state a similar theorem for induced subgraphs, we need a proper modification of $H\left(x_{1}, \ldots, x_{r}\right)$ : we say that a graph $X$ is of type $H\left(x_{1}, \ldots, x_{r}\right)$, if $X$ is obtained from $H\left(x_{1}, \ldots, x_{r}\right)$ by adding some (possibly zero) edges within the sets $V_{u}, u \in V(H)$.

Theorem 2 Let $2 \leq r \leq n,(\ln n)^{-1 / r^{2}} \leq c \leq 1 / 4, H$ be a graph of order $r$, and $G$ be $a$ graph of order $n$. If $G$ contains more than $\mathrm{cn}^{r}$ induced copies of $H$, then $G$ contains an induced subgraph of type $H(s, \ldots s, t)$, where $s=\left\lfloor c^{r^{2}} \ln n\right\rfloor$ and $t>n^{1-c^{r-1}}$. 


\section{Remarks}

- The relations between $c$ and $n$ in Theorems 1 and 2 need some explanation. First, for fixed $c$, they show how large must be $n$ to get valid conclusions. But, in fact, the relations are subtler, for $c$ itself may depend on $n$, e.g., letting $c=1 / \ln \ln n$, the conclusions are meaningful for sufficiently large $n$.

- Note that, in Theorems 1 and 2, if the conclusion holds for some $c$, it holds also for $0<c^{\prime}<c$, provided $n$ is sufficiently large.

- The exponent $1-c^{r-1}$ in Theorems 1 and 2 is not the best one, but is simple.

- Using random graphs, it is easy to see that most graphs on $n$ vertices contain substantially many copies of any fixed graph, but contain no complete bipartite subgraphs with both parts larger than $C \log n$, for some $C>0$, independent of $n$. Hence, Theorems 1 and 2 are essentially best possible.

\section{General notation}

Our notation follows [1]; thus, given a graph $G$, we write:

- $V(G)$ for the vertex set of $G$;

- $E(G)$ for the edge set of $G$ and $e(G)$ for $|E(G)|$;

- $K_{2}$ for the complete graph of order 2 ;

- $K_{2}(s, t)$ for the complete bipartite graph with parts of size $s$ and $t$;

- $\left.f\right|_{X}$ for the restriction of a map $f$ to a set $X$.

\section{Specific notation}

Suppose that $G$ and $H$ are graphs, and let $X$ be an induced subgraph of $H$.

- We write $H(G)$ for the set of injections $h: V(H) \rightarrow V(G)$, such that $\{u, v\} \in E(H)$ if and only if $\{h(u), h(v)\} \in E(G)$.

- We say that $h \in H(G)$ extends $g \in X(G)$, if $g=\left.h\right|_{V(X)}$.

Suppose that $M \subset H(G)$.

- We let

$$
X(M)=\{g:(g \in X(G)) \& \text { (there exists } h \in M \text { extending } g)\} .
$$

- For every $g \in X(M)$, we let

$$
d_{M}(g)=\mid\{h:(h \in M) \&(h \text { extends } g)\} \mid .
$$

Suppose that $Y$ is a subgraph of $G$ of type $H\left(s_{1}, \ldots, s_{r}\right)$ and let $s=\min \left\{s_{1}, \ldots, s_{r}\right\}$. 
- We say that $M$ covers $Y$ if:

(a) for every edge $i j$ going across vertex classes of $Y$, there exists $h \in M$ mapping some edge of $H$ onto $i j$;

(b) there exists $h_{1}, \ldots, h_{s} \in M$, such that $h_{i}(H) \cap h_{j}(H)=\varnothing$ for $i \neq j$, and for all $i \in[s], h_{i}(H)$ contains a vertex from each vertex class of $Y$.

Condition (b) implies that if $M$ covers $Y$, then $Y$ contains $s$ disjoint images of $H$, which are mapped via injections from $M$ and which contain exactly one vertex from each vertex class of $Y$. This technicality is needed for a proof by induction.

\section{Proofs}

The proofs of Theorems 1 and 2 are almost identical, so we shall present only the proof of Theorem 2, for it needs more care. We deduce Theorem 2 from the following technical statement.

Theorem 3 Let $2 \leq r \leq n,(\ln n)^{-1 / r^{2}} \leq c \leq 1 / 4, H$ be a graph of order $r$, and $G$ be a graph of order $n$. If $M \subset H(G)$ and $|M| \geq c n^{r}$, then $M$ covers an induced subgraph of type $H(s, \ldots s, t)$ with $s=\left\lfloor c^{r} 4^{-r^{2}+r} \ln n\right\rfloor$ and $t>n^{1-c^{r-1}}$.

To see that Theorem 3 implies Theorem 2, note that to each induced copy of $H \subset G$ corresponds an injection $h \in H(G)$, and to different copies correspond different injections. Hence, if $G$ contains $c n^{r}$ induced copies of $H$, we have a set $M \subset H(G)$ with $|M| \geq$ $c n^{r}$. By Theorem 3, G contains an induced subgraph $Y$ of type $H(s, \ldots s, t)$ with $s=$ $\left\lfloor c^{r} 4^{-r^{2}+r} \ln n\right\rfloor$ and $t>n^{1-c^{r-1}}$; now Theorem 2 follows, in view of $c^{r} 4^{-r^{2}+r} \geq c^{r^{2}}$.

In turn, the proof of Theorem 3 is based on the following lemma.

Lemma 4 Let $F$ be a bipartite graph with parts $A$ and $B$. Let $2 \leq r \leq n,(\ln n)^{-1 / r^{2}} \leq$ $c \leq 1 / 2,|A|=m,|B|=n$, and $s=\left\lfloor c^{r} 4^{-r^{2}+r} \ln n\right\rfloor$. If $s \leq\left(c / 2^{r}\right) m+1$ and $e(F) \geq$ $\left(c / 2^{r-1}\right)$ mn, then $F$ contains a $K_{2}(s, t)$ with parts $S \subset A$ and $T \subset B$ such that $|S|=s$ and $|T|=t>n^{1-c^{r-1}}$.

\section{Proof Let}

$$
t=\max \left\{x: \text { there exists } K_{2}(s, x) \subset F \text { with part of size } s \text { in } A\right\} .
$$

For any $X \subset A$, write $d(X)$ for the number of vertices joined to all vertices of $X$. By definition, $d(X) \leq t$ for each $X \subset A$ with $|X|=s$; hence,

$$
t\left(\begin{array}{c}
m \\
s
\end{array}\right) \geq \sum_{X \subset A,|X|=s} d(X)=\sum_{u \in B}\left(\begin{array}{c}
d(u) \\
s
\end{array}\right) .
$$


Following [2], p. 398, set

$$
f(x)=\left\{\begin{array}{cc}
\left(\begin{array}{l}
x \\
s
\end{array}\right) & \text { if } x \geq s-1 \\
0 & \text { if } x<s-1
\end{array}\right.
$$

and note that $f(x)$ is a convex function. Therefore,

$$
\sum_{u \in B}\left(\begin{array}{c}
d(u) \\
s
\end{array}\right)=\sum_{u \in B} f(d(u)) \geq n f\left(\frac{1}{n} \sum_{u \in B} d(u)\right)=n\left(\begin{array}{c}
e(F) / n \\
s
\end{array}\right) \geq n\left(\begin{array}{c}
c m / 2^{r-1} \\
s
\end{array}\right) .
$$

Combining this inequality with (1), and rearranging, we find that

$$
\begin{aligned}
t & \geq n \frac{\left(c m / 2^{r-1}\right)\left(c m / 2^{r-1}-1\right) \cdots\left(c m / 2^{r-1}-s+1\right)}{m(m-1) \cdots(m-s+1)}>n\left(\frac{c m / 2^{r-1}-s+1}{m}\right)^{s} \\
& \geq n\left(\frac{c}{2^{r}}\right)^{s} \geq n\left(e^{\ln \left(c / 2^{r}\right)}\right)^{c^{r} 4^{-r^{2}+r} \ln n}=n^{1+c^{r} 4^{-r^{2}+r} \ln \left(c / 2^{r}\right)} .
\end{aligned}
$$

Since $c / 2^{r} \leq 1 / 8<1 / e$ and $x \ln x$ is decreasing for $0<x<1 / e$, and in view of

$$
\frac{2^{2 r^{2}-2 r-1}}{r+1} \geq 1 \geq \ln 2
$$

we see that

$$
\begin{aligned}
c 4^{-r^{2}+r} \ln \left(c / 2^{r}\right) & \geq\left(\left(c / 2^{r}\right) \ln \left(c / 2^{r}\right)\right) 2^{-2 r^{2}+3 r} \geq-\left(2^{-r+1}(r+1)\right) 2^{-2 r^{2}+3 r} \\
& \geq-(r+1) 2^{-2 r^{2}+2 r+1} \ln 2 \geq-1 .
\end{aligned}
$$

Now, $c^{r} 4^{-r^{2}+r} \ln \left(c / 2^{r}\right) \geq-c^{r-1}$ and so,

$$
t>n^{1+c^{r} 4^{-r^{2}+r} \ln \left(c / 2^{r}\right)} \geq n^{1-c^{r-1}} .
$$

Proof of Theorem 3 Let $M \subset H(G)$ satisfy $|M| \geq c n^{r}$. To prove that $M$ covers an induced subgraph of type $H(s, \ldots s, t)$ with $s=\left\lfloor c^{r} 4^{-r^{2}+r} \ln n\right\rfloor$ and $t>n^{1-c^{r-1}}$ we shall use induction on $r$.

Assume $r=2$ and let $A$ and $B$ be two disjoint copies of $V(G)$. We can suppose that $H=K_{2}$, as otherwise we can apply the subsequent argument to the complement of $G$.

Let us define a bipartite graph $F$ with parts $A$ and $B$, joining $u \in A$ to $v \in B$ if $u v \in M$. Set $s=\left\lfloor\left(c^{2} / 16\right) \ln n\right\rfloor$ and note that $s \leq(c / 4) n+1$. Since $e(F)=|M| \geq c n^{2}>(c / 2) n^{2}$, Lemma 4 implies that $F$ contains a $K_{2}(s, t)$ with $t>n^{1-c}$. Hence $M$ covers an induced graph of type $K_{2}(s, t)$, proving the assertion for $r=2$. Now let $r>2$ and assume the assertion true for $r-1$.

Let $V(H)=\left\{v_{1}, \ldots, v_{r}\right\}$ and $H^{\prime}=H\left[\left\{v_{1}, \ldots, v_{r-1}\right\}\right]$.

We first show that there exists $L \subset M$ with $|L|>(c / 2) n^{r}$ such that $d_{L}(h)>(c / 2) n$ for all $h \in H^{\prime}(L)$. Indeed, set $L=M$ and apply the following procedure. 
While there exists an $h \in H^{\prime}(L)$ with $d_{L}(h) \leq(c / 2) n$ do Remove from $L$ all members extending $h$.

When this procedure stops, we have $d_{L}(h)>(c / 2) n$ for all $h \in H^{\prime}(L)$, and also

$$
|M|-|L| \leq \frac{c}{2} n\left|H^{\prime}(M)\right|<\frac{c}{2} n \cdot n^{r-1},
$$

giving $|L|>(c / 2) n^{r}$, as claimed.

Since $H^{\prime}(L) \subset H^{\prime}(G)$ and

$$
\left|H^{\prime}(L)\right| \geq|L| / n>(c / 2) n^{r} / n=(c / 2) n^{r-1},
$$

the induction assumption implies that $H^{\prime}(L)$ covers an induced subgraph $R \subset G$ of type $H^{\prime}(p, \ldots, p)$ with $p=\left\lfloor(c / 2)^{r-1} 4^{-(r-1)^{2}+r-1} \ln n\right\rfloor$. Here we use the inequalities

$$
n^{1-c^{r-2}} \geq n^{1-c} \geq n^{1 / 2}>2^{-4} \ln n \geq(c / 2)^{r-1} 4^{-(r-1)^{2}+r-1} \ln n .
$$

Write $U_{1}, \ldots, U_{r-1}$ for the vertex classes of $R$. Since $H^{\prime}(L)$ covers $R$, we know that there exist $h_{1}, \ldots, h_{p} \in H^{\prime}(L)$ such that $h_{1}\left(H^{\prime}\right), \ldots, h_{p}\left(H^{\prime}\right)$ are disjoint subgraphs of $R$ containing a vertex from $U_{i}$, for all $i \in[r-1]$. For every $i \in[p]$, let

$$
W_{i}=\left\{v:\left(\text { there exists } g \in L \text { extending } h_{i}\right) \&\left(g\left(v_{r}\right)=v\right)\right\} .
$$

That is to say, each vertex in $W_{i}$ together with the vertices of $h_{i}\left(H^{\prime}\right)$ induces a copy of $H$.

Write $d$ for the degree of $v_{r}$ in $H$ and note that each $v \in W_{i}$ is joined to exactly $d$ vertices of $h_{i}\left(H^{\prime}\right)$. Since by our selection, $\left|W_{i}\right|=d_{L}\left(h_{i}\right) \geq(c / 2) n$ for all $i \in[p]$, there is a set $X_{i} \subset W_{i}$ with

$$
\left|X_{i}\right| \geq(c n / 2) /\left(\begin{array}{c}
r-1 \\
d
\end{array}\right) \geq \frac{c n}{2^{r-1}}
$$

such that all vertices of $X_{i}$ have the same $d$ neighbors in $h_{i}\left(H^{\prime}\right)$. Let $Y_{i} \subset[r-1]$ be defined as

$$
Y_{i}=\left\{j: U_{j} \text { contains a neighbor of a vertex in } X_{i}\right\} .
$$

Each of the sets $Y_{1}, \ldots, Y_{p}$ is a $d$-element subset of $[r-1]$; by the pigeonhole principle, there exists a set $A \subset[p]$ with

$$
|A| \geq p /\left(\begin{array}{c}
r-1 \\
d
\end{array}\right) \geq\left\lceil p / 2^{r-2}\right\rceil
$$

such that the sets $Y_{i}$ are the same for all $i \in A$. Note that for every $i \in A$ and every $v \in X_{i}$, the neighbors of $v$ in $h_{i}\left(H^{\prime}\right)$ belong exactly to the same $d$ vertex classes of $R$. Letting $m=\left\lceil p / 2^{r-2}\right\rceil$, we may and shall assume that $|A|=m$.

Let us define a bipartite graph $F$ with parts $A$ and $B=V(G)$, joining $i \in A$ to $v \in B$ if $v \in X_{i}$. Since $\left|X_{i}\right|>c n / 2^{r-1}$ for all $i \in A$, we see that

$$
e(F)>\frac{c}{2^{r-1}} m n .
$$


Also, setting $s=\left\lfloor c^{r} 4^{-r^{2}+r} \ln n\right\rfloor$, we find that

$$
\begin{aligned}
s & \leq c^{r} 4^{-r^{2}+r} \ln n=\left(c 2^{-3 r-3}\right)\left((c / 2)^{r-1} 4^{-(r-1)^{2}+r-1}\right) \ln n \\
& <\left(c 2^{-2 r-2}\right)\left\lfloor(c / 2)^{r-1} 4^{-(r-1)^{2}+r-1} \ln n\right\rfloor+1 \leq\left(c / 2^{r}\right)\left(p / 2^{r-2}\right)+1 \\
& \leq\left(c / 2^{r}\right) m+1 .
\end{aligned}
$$

By Lemma 4, $F$ contains a complete bipartite graph $K_{2}(s, t)$ with parts $S \subset A$ and $T \subset B=V(G)$ such that $|S|=s$ and $|T|=t>n^{1-c^{r-1}}$.

Let $G^{\prime}=G\left[\cup_{i \in S} h_{i}\left(H^{\prime}\right)\right]$ and $G^{\prime \prime}=G\left[\cup_{i \in S} h_{i}\left(H^{\prime}\right) \cup T\right]$. Note that $G^{\prime}$ is an induced subgraph of $R$ and so $G^{\prime}$ is of type $H^{\prime}(s, \ldots, s)$. To prove that $G^{\prime \prime}$ is of type $H(s, \ldots, s, t)$ select $v \in T$ and $h \in L$ such that $\left.h\right|_{V\left(H^{\prime}\right)}=h_{1}$ and $h\left(v_{r}\right)=v$. By our construction $v$ has exactly $d$ neighbors in $h_{1}\left(H^{\prime}\right)$, belonging say to the vertex classes $U_{1}, \ldots, U_{d}$. Since all neighbors of $v$ in $G^{\prime}$ belong to the same vertex classes, and $v$ has $d$ neighbors in each $h_{2}\left(H^{\prime}\right), \ldots, h_{s}\left(H^{\prime}\right)$, we see that $v$ is joined to every vertex in $\cup_{i=1}^{d} U_{i}$, and is not joined to any vertex in $V\left(G^{\prime}\right) \backslash\left(\cup_{i=1}^{d} U_{i}\right)$. Since this holds for all vertices $v \in T$, we see that $G^{\prime \prime}$ is of type $H(s, \ldots, s, t)$.

To finish the proof, we shall show that $L$ covers $G^{\prime \prime}$. By the induction assumption, $L$ covers $R$, hence for every edge $i j$ going across vertex classes of $G^{\prime}$, there exists $h \in L$ mapping some edge of $H$ onto $i j$. On the other hand, let $u \in h_{i}\left(H^{\prime}\right)$ be joined to $v \in T$; by our construction there exist $h \in L$ such that $\left.h\right|_{V\left(H^{\prime}\right)}=h_{i}$ and $h\left(v_{r}\right)=v$. Thus, $h^{-1}(u) v \in E(H)$, and $h$ maps an edge of $H$ onto $u v$. This proves condition (a) for covering.

Finally, taking $s$ distinct vertices $u_{1}, \ldots, u_{s} \in T$, by the construction of $T$, for every $i \in S$, there exists $g_{i} \in L$ with $\left.g_{i}\right|_{V\left(H^{\prime}\right)}=h_{i}$ and $g_{i}\left(v_{r}\right)=u_{i}$. Hence, $L$ covers $G^{\prime \prime}$, completing the induction step and the proof of Theorem 3.

\section{References}

[1] B. Bollobás, Modern Graph Theory, Graduate Texts in Mathematics, 184, SpringerVerlag, New York (1998).

[2] L. Lovász, Combinatorial problems and exercises, North-Holland Publishing Co., Amsterdam-New York (1979).

[3] V. Nikiforov, Graphs with many $r$-cliques have large complete $r$-partite subgraphs, to appear in Bull. London Math. Soc. 